\title{
Reductionism And Holism In Allergy
}

\author{
Pandey $P^{l}$ \\ Address for correspondence \\ Dr. Prajwal Pandey \\ Senior Resident \\ Department of Dermatology \\ BPKIHS, Dharan, Nepal
}

${ }^{1}$ Senior Resident, Department of Dermatology, BP Koirala Institute of Health Sciences, Dharan

Since Descartes and the Renaissance, science, including medicine, has taken a distinct path in its analytical evaluation of the natural world. This approach can be described as "divide and conquer," and it is rooted in the assumption that complex problems are solvable by dividing them into smaller, simpler, and thus more tractable units. Because the processes are "reduced" into more basic units, this approach has been termed "reductionism" and has been the predominant paradigm of science over the past two centuries. Reductionism pervades the medical sciences and affects the way we diagnose, treat, and prevent diseases. While it has been responsible for tremendous successes in modern medicine, there are limitations to reductionism.

While the implementation of clinical medicine is systems-oriented, the science of clinical medicine is fundamentally reductionist.Though this approach is easily executable in clinical practice and its success is undeniable, it leaves little room for contextual information. The disease, and not the person affected by it, becomes the central focus and it neglects the complex interplay between disease and treatment. Our contemporary analytical tools are simply not designed to address more complex questions, and, thus, questions such as "how do a person's sleeping habits, diet, living condition, comorbidities, and stress collectively contribute to his/her health?" remain largely unanswered.

Technical and moral fragmentations are bound to occur when an issue is broken into separate parts and those parts studied separately. For example, the study of allergies as a consequence of exposure to mold is largely the province of doctors interested in respiratory medicine; identifying mold found in houses has been confined to building research and microbiology; measuring dampness in dwellings has been the task of Environmental Health Officers and surveyors; and investigating the reasons for the damp has been the concern of architects. These groups of experts rarely work together or even pool their findings. Moreover, pointing to social factors may be regarded as a political act that lies outside the province of medical jurisdiction. Links between social problems and ill health, which properly belong in the realm of public health, are thus reduced to the level of clinical medicine.

An approach of 'general susceptibility' is based on the hypothesis that people may become vulnerable to a variety of ills because of the social and economic strains under which they live and is not limited to either the poorer or the more affluent population. The current medical model and the general susceptibility model are by no means incompatible. The treatment of individuals does nothing to alter the conditions that gave rise to the disorder in the first place. Indeed a person may be successfully diagnosed and treated, only to return to precisely the situation that created the problem in the first place.

The alternative explanation that has received much recent attention, due to systems biology, is the systems perspective. Rather than dividing a complex problem into its component parts, the systems perspective appreciates the holistic and 


\section{Letter to Editor}

composite characteristics of a problem and evaluates the problem with the use of computational and mathematical tools. The systems perspective is rooted in the assumption that the forest cannot be explained by studying the trees individually.

The science underlying our medical practices, from diagnosis to treatment to prevention, is based on the assumption that information about individual parts is sufficient to explain the whole. But there are circumstances in which the complex interplay between parts yields a behavior that cannot be predicted by the investigation of the parts alone. The failure to account for these circumstances is the common denominator for the explanations of why the current practices are, in many cases, inadequate. A relatively new branch of science called systems biology was conceived to address the molecular complexities seen in biological systems. ${ }^{1,2}$ One major impetus for its creation was the human genome project. The human genome contains more than 30,000 genes. ${ }^{3}$ The richness of information is derived not only in the genes themselves but also in the interaction between genes and between their respective products. Between each hierarchical level, modifications (e.g., alternative splicing) are made, and at each hierarchical level (e.g., transcription), thousands of molecules interact with other molecules to create a complex regulatory network. What becomes evident from these molecular analyses is that phenotypic traits emerge from the collective action of multiple individual molecules. ${ }^{4,5}$ Therefore, the previous notion that a single genetic mutation is responsible for most phenotypic defects is overly simplistic. Complex diseases such as atopic eczema, asthma, or hypersensitivity cannot generally be explained by a single genetic mutation.

\section{References}

1. Kitano H. Systems biology: A brief overview. Science 2002; 295: 1662-4.

2. Ehrenberg M, Elf J, Aurell E, Sandberg R, Tegner J. Systems biology is taking off. Genome Res 2003; 13: 2377-80.

3. Weston AD, Hood L. Systems biology, proteomics, and the future of health care: Toward predictive, preventative, and personalized medicine. J Proteome Res 2004; 3: 179-96.

4. Guttmacher AE, Collins FS. Genomic medicine-A primer. N Engl J Med 2002; 347: 1512-20.

5. Kauffman S (1993) The origins of order: Self organization and selection in evolution. New York: Oxford University Press. 734 p. 